\title{
Cell manipulation by use of diamond microparticles as handles of optical tweezers
}

\author{
Chi-Kuang Sun and Yin-Chieh Huang \\ Department of Electrical Engineering and Graduate Institute of Electro-Optical Engineering, National Taiwan \\ University, Taipei 10617, Taiwan \\ Ping Chin Cheng \\ Department of Electrical Engineering, State University of New York, Buffalo, New York 14260-2050 \\ Hung-Chi Liu and Bai-Ling Lin \\ Institute of Molecular Biology, Academia Sinica, Taipei 11529, \\ and Molecular and Cell Biology Division, Developement Center for Biotechnology, Taipei 10659, Taiwan
}

Received June 13, 2000; revised manuscript received April 24, 2001

\begin{abstract}
We report the experimental results of our using irregularly shaped diamond microparticles as handles for laser tweezers. Because of their irregular optical shape, control of the rotation of diamond microparticles can easily be achieved in a gradient force optical trap by use of a fixed linearly polarized beam with a fundamental Gaussian mode. By changing the laser focal plane upon a diamond particle near the liquid surface or interfaces, one can fully manipulate both the direction and speed of the rotation. The ability to manipulate a diamondparticle-tagged biological specimen by optical tweezers is discussed. The application of these particles as handles for optical tweezers is demonstrated by optical manipulation of biological cells. Independent movement of linear translation and rotation, with controllable rotation directions and speeds, is successfully achieved. (C) 2001 Optical Society of America
\end{abstract}

OCIS codes: $174.520,170.4520,147.010,140.7010$.

\section{INTRODUCTION}

The development of the gradient force optical trap, called optical tweezers, has made it possible to manipulate biological materials with a single beam of laser light. ${ }^{1,2}$ In a strongly focused optical field, an optical trap can produce forces on trapped particles in the range of tens to hundreds of piconewtons. Because of the field gradient, such a force is sufficient to overcome forces that are due to both gravity and radiation pressure and to arrest the movement of microscopic particles, including active biological cells. These laser tweezers can be applied to the manipulation of biological materials, making the best use of the essentially noninvasive feature of light. Piconewtons are also well matched to the level of forces encountered in biological ultrastructures. Forces of this magnitude can move cells, bend cytoskeletal elements, distort proteolipid membranes, arrest organelle movements or swimming bacteria, and overcome the motion of biological motors. For application in the study of biophysical properties, one especially powerful approach has been to use micrometersized spheres such as silica or latex beads, as handles to assist in the manipulation of cells or even of molecules and molecular assemblies with surface coatings such as gelsolin and kinesin. ${ }^{3-6}$ This approach can minimize possible photodamage by preventing direct illumination of biological tissues and provide controllable motion with calibrated forces for detailed biophysical studies. By us- ing a spherically shaped bead, however, one can achieve only translation movement in an optical tweezers arrangement. No rotational controllability can be achieved by use of fixed linearly polarized laser light with a fundamental Gaussian transverse mode.

Several techniques have been proposed and demonstrated for achievement of rotational control in an optical trap. Particle rotation was achieved by partial absorption of laser light with a circular polarization or with a high-order Laguerre-Gaussian mode, ${ }^{7,8}$ which resulted in the transfer of spin or orbital angular momentum. By using high-order $\mathrm{TEM}_{0 n}$ mode laser beams one found that the long axis of an elliptical cell surface aligns with the elongated direction of the high-order beam distribution. Cell rotation can be demonstrated by a $360^{\circ}$ rotation of this laser beam about its optical axis. ${ }^{9}$ Birefringent microobjects were found to be rearrangable through optically induced angular alignment by a linearly polarized light. ${ }^{10}$ It was shown that rotating the laser polarization could rotate birefringent calcite particles. ${ }^{11}$ Changing the laser polarization into elliptical polarization allows the irregularly shaped birefringent calcite particles to spin with constant rotational frequency. ${ }^{11}$ By irradiating two trapping laser beams independently of the two ends of an object, one also achieved rotation by fixing one laser beam while scanning the other beam circularly in the sample plane. ${ }^{12} \mathrm{~A}$ windmill-type rotation of combined 
Mie particles caused by optical forces of the evanescent field generated at the surface of a gadolinium gallium garnet prism was recently demonstrated. ${ }^{13}$ Optically induced rotation was also facilitated by use of objects that were specially fabricated to have rotational symmetry or asymmetry, ${ }^{14-18}$ for which case their application in the field of micromotors with possible applications in biological studies was emphasized. ${ }^{16,18}$ However, most methods require a relatively complicated experimental setup, for instance, including a polarization-control component or a laser mode control component, to achieve and control the rotation of a high-order laser mode or laser polarization. These experiments are focused on elliptical, birefringent, or specially engineered objects. Some methods require absorption of laser light, which will result in cell damage in biological applications. The rotational directions of the specially engineered objects previously demonstrated $^{14-18}$ were determined by the objects' designed shape, which did not permit variation of the rotational direction. Recently there were several reports of selfrotation of irregularly shaped particles in a laser trap with a fixed linearly polarized light. ${ }^{19,20}$ The selfrotation was caused by the torque that resulted from the total forces' acting on the particle surface. The rotation speed was found to be linearly dependent on the irradiated beam power. The rotation rate was controlled by a feedback loop connecting the measured rotation rate and the trapping beam power with a proposed application as micromotors and microfans in microelectromechanical systems.

In this paper we investigate the application of irregularly shaped microparticles as the handles of optical tweezers for rotational control of biological specimens. The ability to rotate a cell in a culture medium is important in biology. Cells frequently have structural orientation (polarity) that determines the physiology and function of the cell. Even a spherically shaped cell has polarity (e.g., in terms of membrane potential or membrane protein distribution). Therefore, in a biological experiment the capability to orient the cell under a microscope is important. Our cell rotation method allows easy access to cell organelles for microinjection, microdissection, and signal recording in physiological research. This rotation capability is also important for studying the behavior of twisting macromolecules and biological enzyme motors. ${ }^{4-6}$ The use of a handle to manipulate the cell is preferable to direct manipulation of the cell with optical tweezers. Recent results have shown that, under intense near-infrared illumination at an intensity comparable with that used in typical tweezing experiments, ultrastructural damages in mitochondria have been detected. ${ }^{21,22}$ In addition, effects on cell motility and cloning have been reported. ${ }^{21,22}$ In plant cells, rapid changes in chloroplast autofluorescence owing to near-infrared direct excitation were reported recently. $^{23}$ The self-rotation effects of irregularly shaped particles make rotational control easy and compatible with most optical tweezers by use of a strongly focused laser beam with a fundamental Gaussian mode and a fixed linear polarization with no need for most biologist users to continually change the polarization. In addition, the simple optical configuration provides easy setup in a standard biological laboratory.
We perform our studies by using diamond microparticles that are readily available in the lapping industry. The particles are not only transparent at visible and infrared wavelengths and stable under intense laser light but are also biologically inert. The ionic strength of diamond can withstand the regular culture medium, which is similar to seawater. With a biologically inert surface, diamond particles will not release any bioactive ionic compound such as magnesium or calcium; the latter is a secondary messenger involved in most physiological processes in a concentration range of $10^{-5}$ to $10^{-7} \mathrm{M}$. Inasmuch as strict control of the compound (such as $\mathrm{Ca}^{++}$) concentration in the culture medium is routine in biological science, the use of diamond particles is highly desirable because of the particles' lack of biological activity. Cell attachment to a diamond particle can easily be achieved by various well-established methods (e.g., the use of polyamino acids such as polylysine, and natural peptides). With a wide bandgap, a diamond particle can avoid not only single-photon absorption but also multiphoton absorption under focused laser light at either visible or infrared wavelengths, thus reducing absorptioninduced heating and damage.

Even though the self-rotation effect can induce rotation of diamond particles in an optical trap with fixed polarization, full rotational control also requires control of the rotational direction or even stopping the rotation while the particle remains trapped to enable linear translation and rotation of a specimen to be performed independently. Because diamond is not a birefringent material, control of its rotation cannot be accomplished by control of the polarization of the laser's light. We achieve the ability to control rotation by changing the laser's focal plane near the liquid surface or interfaces. Control of both the rotational direction and the angular speed were successfully achieved. These diamond microparticles can thus serve as optical handles for a laser tweezers, providing more rotational control than regular spherical beads, with the advantage that a conventional laser trapping setup can be used without any modification. Their application as handles of optical tweezers is demonstrated by optical manipulation of biological cells. Independent movement of linear translation and rotation, with controllable rotation directions and speeds, is achieved.

\section{MATERIALS AND METHODS}

\section{A. Sample Preparation}

The leaves of 4- to 5-week-old Arabidopsis thaliana (ecotype Columbia) were used for protoplast preparation. The mesophyll protoplasts were isolated by enzyme digestion to remove the cell walls and were incubated in an isotonic culture medium (containing inorganic salts, vitamins, organic acids, $0.06 \mathrm{M}$ sucrose, $0.31 \mathrm{M}$ glucose, and morpholineethanesulfonic acid; ph 5.6), as described by Huang and Chen. ${ }^{24}$ Diamond microparticles were obtained from General Electrical Superabrasives (Worthington, Ohio). We used 15-25- $\mu$ m-diameter irregularly shaped diamond particles with a refractive index of 2.4 in the experiments. The diamond particles were mixed with $0.01 \%$ poly-L-lysine (Sigma P8920), which promotes adhesion of cells to solid substrates. ${ }^{25}$ After 2-h incuba- 
tion and thorough washing in water, the coated particles were resuspended in the same culture medium. Proper concentrations of the coated diamond particles and mesophyll protoplasts were mixed to permit one-to-one adhesion.

\section{B. Optical Tweezers and Video Acquirement}

Figure 1 shows the experimental setup used for studies of optical trapping and manipulation. A continuous-wave Ti:sapphire laser (Spectra-Physics 3900s), pumped by an all-solid-state frequency-doubled $\mathrm{Nd}: \mathrm{YVO}_{4}$ laser (SpectraPhysics Millennia V), was used as the light source. Because the wavelength used was shorter than that of the laser trap that is used with a Nd:YAG laser, the laser trap constructed with the Ti:sapphire laser had a much stronger trapping capability, with less laser power and no damage to cellular organelles such as mitochondria and mitotic spindles. ${ }^{26}$ The Ti:sapphire laser was operated in the near infrared with a central wavelength of $790 \mathrm{~nm}$ at a bandwidth less than $40 \mathrm{GHz}$. The output beam had a fundamental Gaussian $\mathrm{TEM}_{00}$ mode, with a minimum 100:1 extinction ratio in linear polarization. After it passed through two telescope lenses and a beam splitter, the laser beam was introduced into a conventional optical microscope (Leitz Ergolux) and focused with a Leitz $50 \times$ objective lens (PLAN L50×; $\infty / 0$, N.A. $=0.6$ ) to create an optical trap. A set of telescope lenses was used so the laser beam would be focused on the image plane, and the mode size was optimized to fill the back aperture of the objective lens. The whole sample cell was placed close to the focus of the objective lens and was backlighted with an incandescent source. The specimen was monitored with the same $50 \times$ objective through the beam splitter with an associated tube lens and an infrared-blocking filter into a charged-coupled device (CCD) camera, which was connected to a television monitor. Video sequences were recorded with a frame grabber in a personal computer. Even though the rotation of trapped diamond particles can easily be observed with a CCD camera, video images are unsuitable for automatic and high-spin-rate measurement because of limitations in the video sampling rate. We employed the method described in Ref. 19 to measure the rotation rate by monitoring the temporal

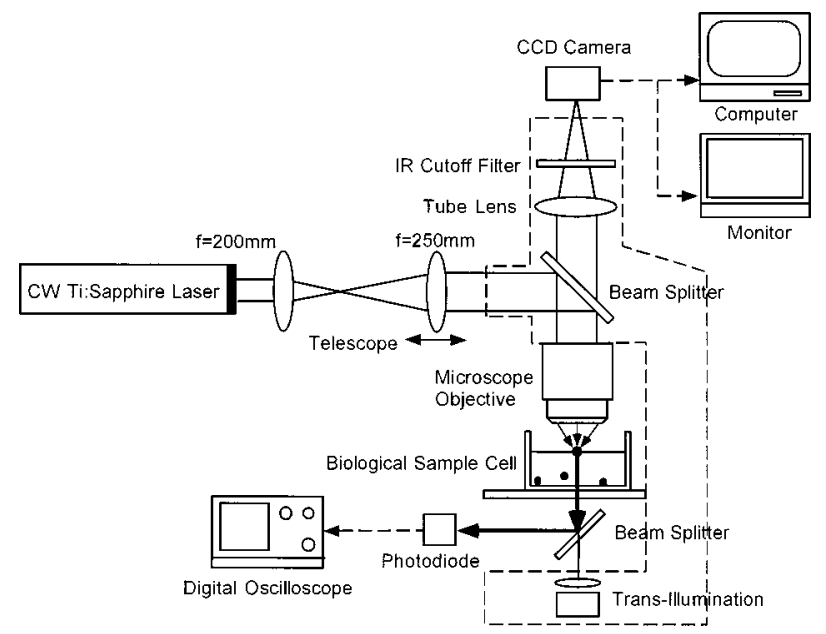

Fig. 1. Apparatus used for optical manipulation of biological cells and diamond microparticles. intensity variation of the scattered light from the diamond particle. The scattered laser light was detected by a photodiode and then output to an oscilloscope. The rotation rate was then derived from the period of the signal variation. Using the scattered laser light with the video image, we estimated that the spot size of the focused laser beam in the trapped diamond particle was $\sim 1 \mu \mathrm{m}$ for our specific wavelength and with the $50 \times$ objective. We applied 50-500 $\mathrm{mW}$ of optical power for the trapping experiments. At least 50 or $100 \mathrm{~mW}$ of power, respectively, was required for successful trapping for linear translation or for manipulating rotation for the 15-25 $\mu \mathrm{m}$-diameter diamond particles with the $50 \times$ objective.

\section{RESULTS AND DISCUSSIONS}

\section{A. Rotational Control of Diamond Particles}

Because the optical refraction and reflection resulted in momentum transfer from the incident light to the particles, the optical beam exerted forces on the particles. The net force could then be described by integration of these forces over the angles at which the light intersects the particles. $^{27}$ Trapping is successfully achieved when the net force is balanced. However, with irregularly shaped objects, the net torque will not necessarily be zero when the net force is balanced. ${ }^{19,20}$ This result is different from that for a spherically shaped object for which the net torque that results from a Gaussian-shaped trapping force is zero because of cancellation by geometric symmetry. In a viscous medium, this net torque $\tau$ will be balanced by the drag torque, $\tau=D \Omega$, where $D$ is the drag coefficient and $\Omega$ is the angular speed. With a constant $D$, the angular speed could thus be found to be linearly proportional to the applied optical intensity, which is also proportional to the net torque. Self-rotation was previously achieved for irregularly shaped nonbirefringent particles with a laser beam that had a fundamental Gaussian mode and a fixed linear polarization, ${ }^{19,20}$ even in a three-dimensional trap in air. ${ }^{20}$

In our experiments, the rotation speeds and directions of the diamond microparticles were affected by particle shape, so they are different for different particles with a fixed laser power. As in previous experiments, ${ }^{19,20}$ the rotation speed of a single diamond particle was linearly dependent on the illuminated laser power. Figure 2 shows the power dependence of the rotation rate for three different diamond microparticles. The rotation rate was measured with an oscilloscope and scattered laser light. The dotted lines are linear fits. A maximum slope value of $41 \mathrm{rpm} / \mathrm{mW}$ was observed for these diamond microparticles. For some particles, their spinning angular velocities were so low that an oscilloscope is not suitable for this measurement. With $150-\mathrm{mW}$ average optical power with the $50 \times$ objective, the spinning speed of a $20-\mu \mathrm{m}-$ diameter diamond particle can vary from $6000 \mathrm{rpm}$ (measured with an oscilloscope) to a low speed of less than 30 rpm (measured with a CCD) with different spinning directions (clockwise or counterclockwise), depending on the particle shape and laser focal position relative to the liquid surface. The rotation direction and speed were, however, found to be the same if we moved the particles with the laser trap laterally. 


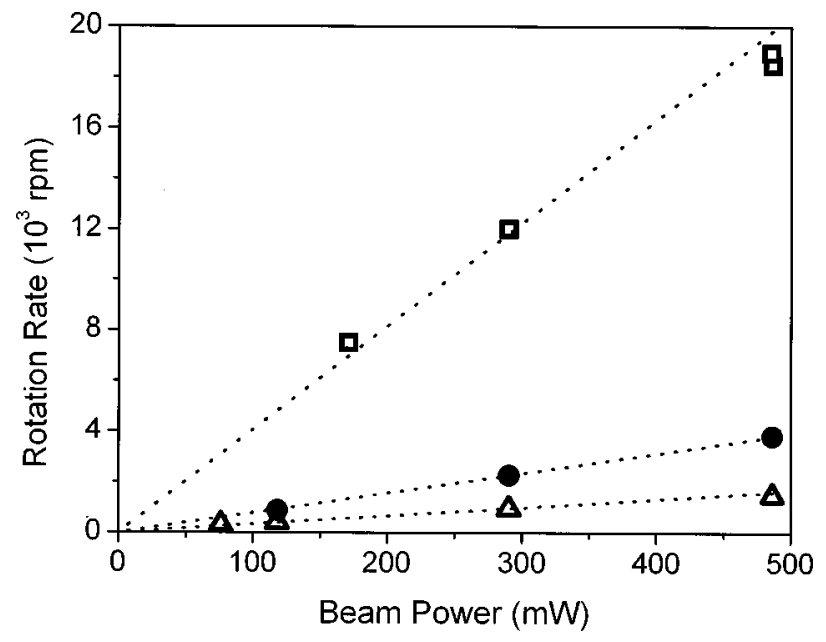

Fig. 2. Rotation speeds of three diamond microparticles versus illuminated laser power with a $50 \times$ objective. The linear dependence on the illuminated laser power can be found. Dotted lines are fits with slopes ranging from 3.3 to $41 \mathrm{rpm} / \mathrm{mW}$.

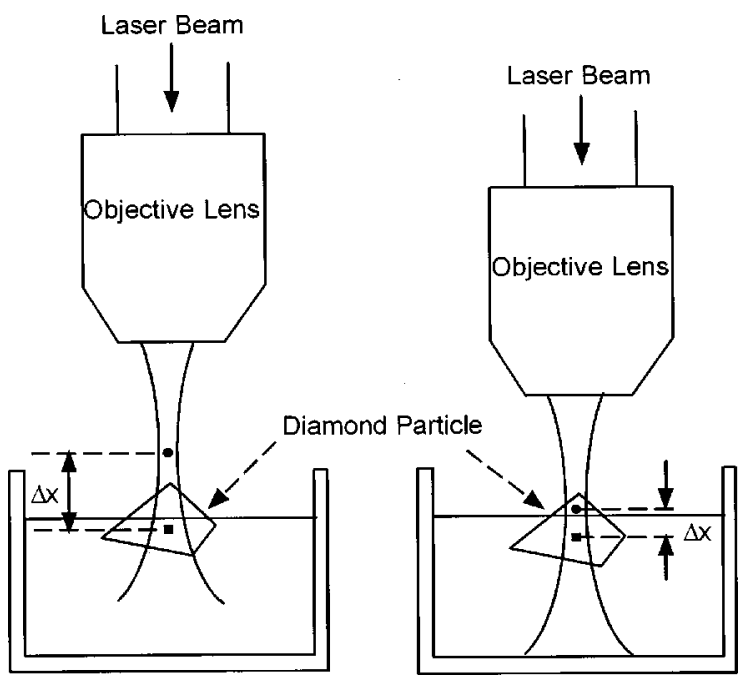

Fig. 3. One can achieve rotational control of an irregularly shaped diamond particle by moving the objective in the laser propagation direction close to the liquid surface or interfaces. When the particle is close to the surface, the change in buoyant forces and surface tensions will modify the relative position $\Delta x$ between the laser's focal point (filled circles) and the center of gravity of the diamond (filled squares) under force equilibrium.

For rotational control of a single diamond particle, including control of both rotational direction and angular speed, not only the beam power but also the relative position between the laser focal point and the center of gravity of the diamond under force equilibrium must vary. However, in a regular arrangement, the center of gravity of the diamond particles will follow the optical trap's position because of the force-equilibrium requirement, and no relative variation in position between the laser's focal point and the particle's center of gravity can be achieved. In this case, the rotation of trapped particles will behave as in the previous air-trapping experiment ${ }^{20}$ in which the silica particle rotation was found to be highly dependent on the particle shape and radiation pressure; the former factor determines the rotation direction, and the latter factor determines the rotation speed. One way to vary the relative position of the laser focal point and the center of gravity of a trapped particle under force equilibrium is to perform the experiments with the particle close to the liquid surface or interfaces, as schematically shown in Fig. 3. When the particle is close to the surface or interface, the change in buoyant forces and surface tensions will modify the relative position in the beam-propagation direction between the laser's focal point and the center of gravity of the diamond under force equilibrium, thus changing the rotational speed and even direction. Al-
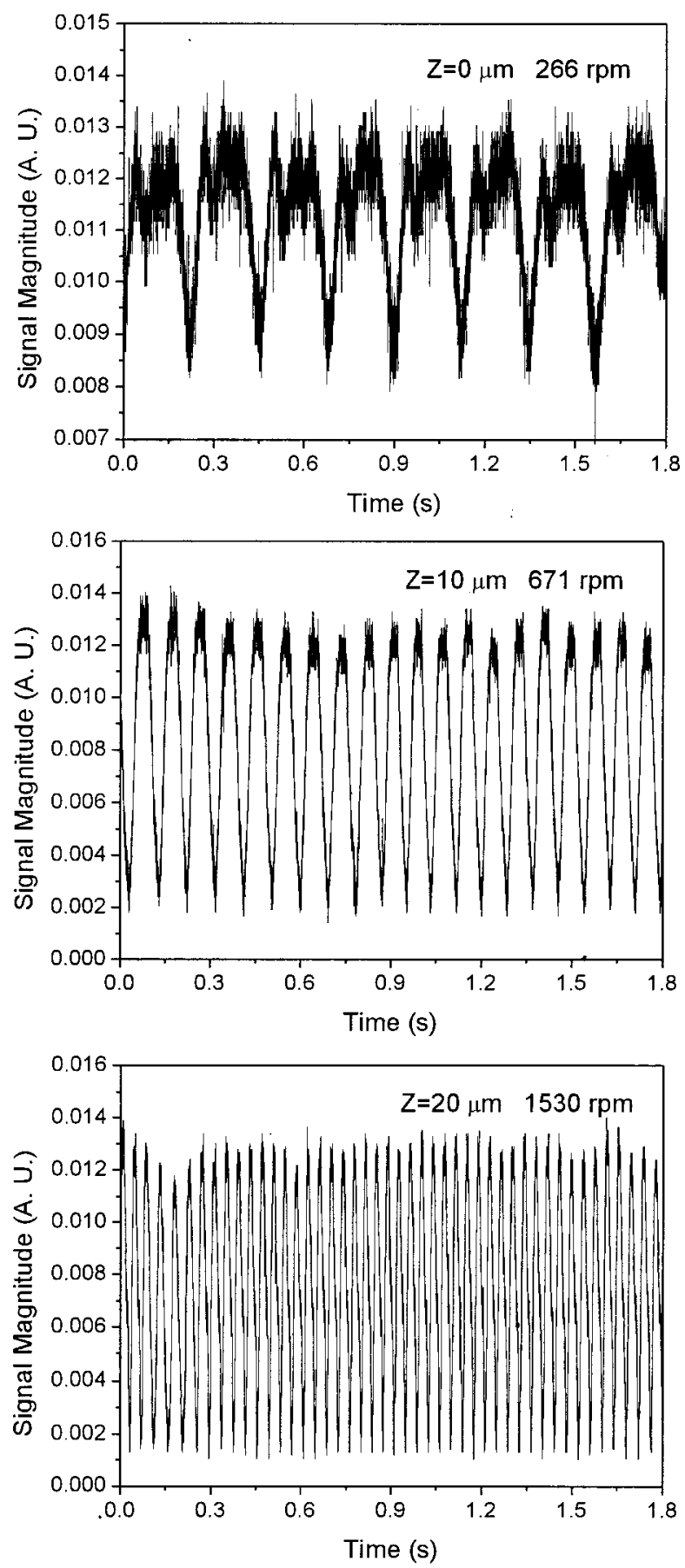

Fig. 4. Periodic detected signals from a rotating diamond microparticle with three focal-plane positions with a fixed laser power of $486 \mathrm{~mW}$. The variation in relative focal-plane position between consecutive traces is $10 \mu \mathrm{m}$. 

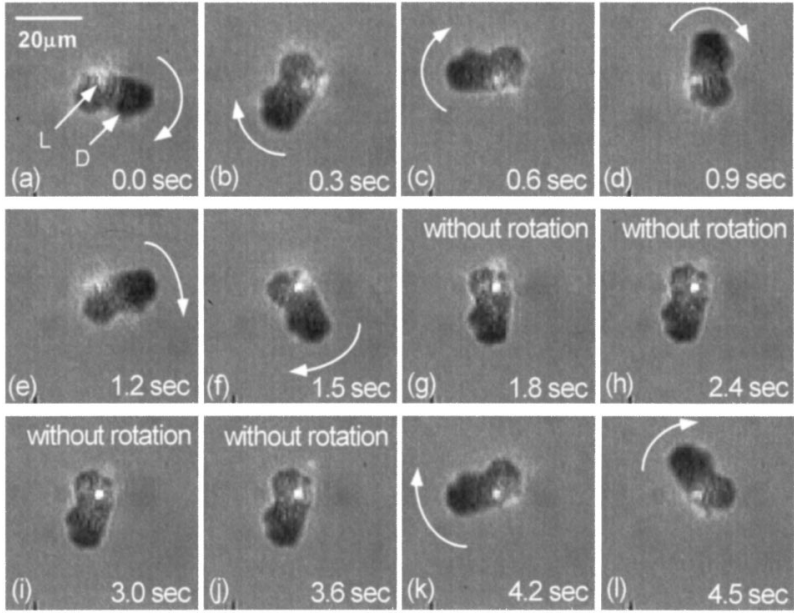

Fig. 5. Micrographs of an optically trapped diamond microparticle. Complete $360^{\circ}$ rotation can be observed. By vertically moving the microscope objective for $1.8 \mathrm{~s}(\mathrm{~g})$ and for $4.2 \mathrm{~s}(\mathrm{k})$, we can observe optical trapping without and with rotation. The arrowheads indicate the directions of rotation. The black object labeled D in (a) is the diamond particle. The scattered laser light is labeled $\mathrm{L}$.
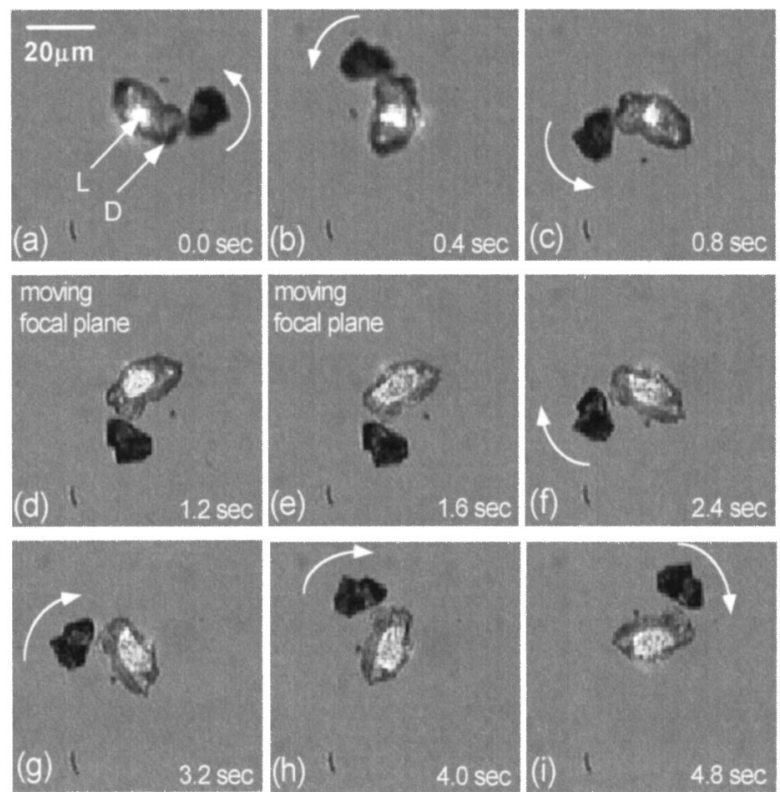

Fig. 6. Micrographs of two optically trapped diamond microparticles. We controlled the rotational direction of optically trapped diamond micro-particles by vertically moving the microscope objective for $\sim 1.0 \mathrm{~s}(\mathrm{~d})$. Different rotation directions can be observed for (a)-(c) (counterclockwise) and (f)-(i) (clockwise).

though the rotation rate is not proportional to the position of the laser focal plane because of the irregularly shaped diamond particles and the complex redistributed equilibrium composed of the laser radiation force, buoyant forces, and surface tensions, the modified rotation rate could be varied as we vertically modified the position of the laser focal point. Figure 4 shows examples of the periodic detected signals from the scattered light of a rotating diamond particle with three different focal-plane positions with a fixed laser power of $486 \mathrm{~mW}$. With a $10-\mu \mathrm{m}$ variation in the position of the focal plane between successive positions at the water surface, drastic angular velocity changes from 1530 to 671 to $266 \mathrm{rpm}$ can be observed. Furthermore, because of the asymmetry of the diamond particle in the beam-propagation axis, we can achieve additional rotational control, especially rotational direction control, by moving the objective in the direction of laser propagation.

Figure 5 shows the result of optical manipulation of the diamond microparticles. The micrographs in Fig. 5 are presented in a time-lapse format; the relevant elapsed time is indicated. The incident optical power intensity was $150 \mathrm{~mW}$. The slices from 0.0 to $1.5 \mathrm{~s}$ show the clockwise rotation of an irregularly shaped diamond particle in the laser trap. The arrowheads point in the direction of rotation. The dark irregularly shaped object [labeled D in Fig. 5(a)] is the diamond particle, and the bright spot inside the diamond particle is the focused laser light observed through scattering (labeled L). The diamond particle appears dark as a result of the refractive-index difference between diamond and water with backilluminated white light. At 1.5-4.5 s, we demonstrate the controllability of the rotational motions. At 1.8 and $3.6 \mathrm{~s}$, respectively, we moved the focal plane to stop and resume the rotational motion. By vertically moving the laser focal plane relative to the liquid surface, we can observe the change in the net torque induced by the optical forces such that the induced rotations can be fully stopped. No rotation was observed at 1.8 to $3.6 \mathrm{~s}$, indicating a balanced net toque. This behavior is different from that of an untrapped particle, which would maintain irregular movement. Independent movements of rotational and linear translation can thus be accomplished.

The effects of moving the focal plane can also be exploited to control rotation direction, based on the redistributed forces upon the irregularly shaped particles. Figure 6 demonstrates how one can control the rotation direction of irregularly shaped diamond particles by vertically moving the objective with a fixed optical power of $150 \mathrm{~mW}$. We have chosen to show two diamond particles in each image to present this effect with better clarity. From 0.0 to $1.2 \mathrm{~s}$, the particles were rotating in the counterclockwise direction. By moving the laser focal plane into the liquid near $1.0 \mathrm{~s}$, we changed the rotation of the diamond particles from its original counterclockwise direction into the clockwise direction. This change in rotation direction was due to the change in the relative positions of the laser's focal plane and the center of gravity of the diamond particles because of the buoyant force and surface tension near the liquid's surface. It is also interesting to observe that some diamond microparticles cannot rotate in both clockwise and counterclockwise directions, probably because of their specific geometries. However, by moving the focal plane about at the liquid surface, we were able to stop the rotational movement of all diamond particles.

\section{B. Using Diamond Particles As Optical Handles}

After adhesion of the diamond particles to cells and molecular assemblies, the irregularly shaped diamond particles can serve as optical handles in an optical tweezers setup for rotation as well as translation manipulations of biological specimens. The concept is demonstrated with 

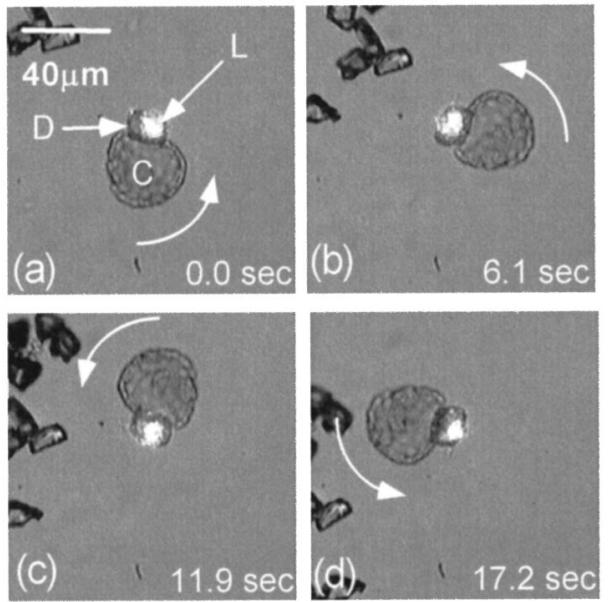

Fig. 7. Micrographs of a diamond-particle-tagged mesophyll protoplast. Rotation of $360^{\circ}$ about the optical axis can be achieved in a regular tweezers setup. The black object labeled D in (a) is the diamond particle. The scattered laser light is labeled $\mathrm{L}$, and the mesophyll protoplast cell is labeled $\mathrm{C}$.
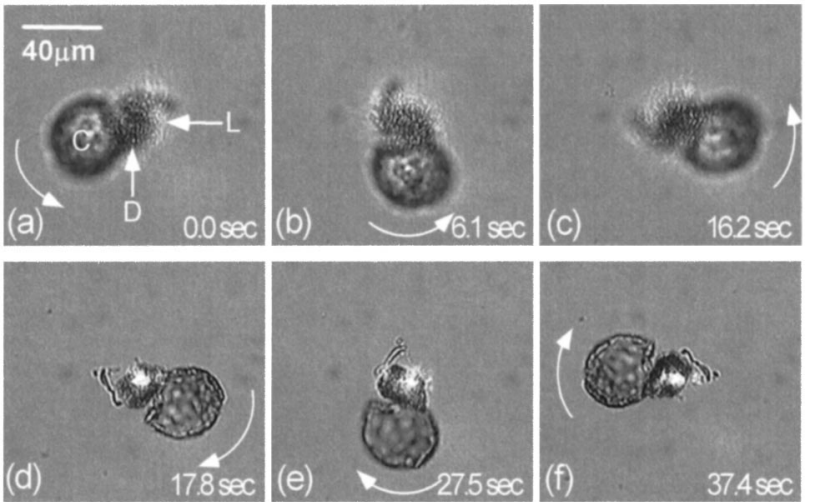

Fig. 8. Micrographs of a diamond-particle-tagged mesophyll protoplast. We controlled the rotation direction of the mesophyll protoplast by vertically moving the microscope objective at $17.0 \mathrm{~s}$ in the time sequence. The arrowheads indicate the directions of rotation. Different rotational directions can be observed for (a)-(c) (counterclockwise) and (d)-(f) (clockwise).
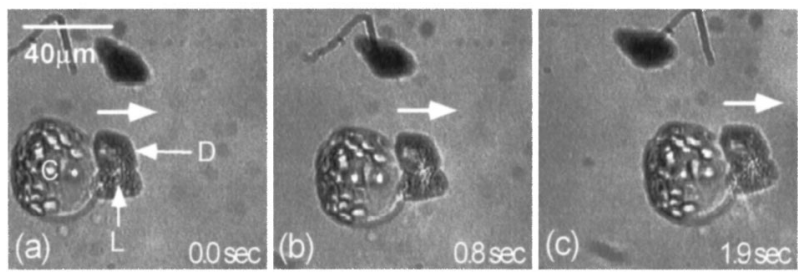

Fig. 9. Micrographs of a diamond-particle-tagged mesophyll protoplast. The arrowheads indicate the direction of movement. Linear translation of a mesophyll protoplast was achieved by use of the irregularly shaped diamond particle as an optical handle.

Arabidopsis thaliana mesophyll protoplasts. Rotational motion can be successfully observed even with protoplasts of $30-50-\mu \mathrm{m}$ diameter adhering to a diamond particle. Figure 7 shows the counterclockwise rotation of a mesophyll protoplast (labeled C) with an irregularly shaped diamond particle (labeled D) used as the optical handle in the laser tweezers setup with $150 \mathrm{~mW}$ of optical power. Full circle $\left(360^{\circ}\right)$ rotation can be successfully achieved. Reduction of the angular speed as a result of increasing rotational inertia of the protoplast was observed. Higher angular speed can be achieved by use of higher incident laser power, a higher-N.A. objective, or a smaller diamond particle. A smaller cell will also increase angular speed because there will be a reduction in drag.

We can control the rotation direction and the angular speed of the cells by moving the laser's focal plane upon the diamond optical handles. Figure 8 demonstrates our control of the rotational direction of a mesophyll protoplast by moving the objective vertically with a fixed optical power of $150 \mathrm{~mW}$. At 0.0 to $16 \mathrm{~s}$, the protoplast was rotating in the counterclockwise direction. By moving the laser focal plane into the liquid near $17.0 \mathrm{~s}$, we changed the rotation direction of the protoplast from counterclockwise to clockwise. It is interesting to notice the different picture qualities for the images with different rotation directions shown in Fig. 8, which are due to different relative positions between the laser's focal plane and the center of gravity of the diamond particle. Figures $8(a)-8(\mathrm{c})$ show the situation when there is a separation between the image plane, which coincides with the laser focal plane, and the particle's center of gravity.

Complete manipulation of cells includes not only rotational but also translational control. Figure 9 shows the linear translation of a mesophyll protoplast with the optical tweezers setup with an irregularly shaped diamond particle used as an optical handle. The arrows indicate the moving direction of the linear translation. As the laser beam moved toward the right-hand side of the image, the diamond-particle-tagged mesophyll protoplast was found to follow the laser-beam-induced trap and to perform a linear translation. This confirms the fact that one can use an irregularly shaped particle to make independent rotational and translational movements of a biological specimen.

\section{SUMMARY}

In summary, we have demonstrated optical manipulation of a biological sample, a plant cell, with a strongly focused laser beam and irregularly shaped diamond microparticles as optical handles. Cell-to-diamond-particle attachment can be facilitated by the use of poly-L-lysine, but this attachment can also be achieved by the use of a receptor-ligand or an antibody-antigen reaction. As a result of our experiments, the optical trapping field is found not only to be able to provide linear translation movements but also to achieve rotation without absorption of light. By changing the laser's focal plane near the liquid surface or interfaces, we can fully manipulate both the rotation direction and speed with fixed linear polarization. For application as optical handles, these diamond microparticles can thus provide more rotational control than can spherical beads and can permit the use of a conventional laser tweezers setup with a fundamental Gaussian laser mode and fixed linear polarization without any sophisticated modifications. The chosen diamond particles also have the advantages of easy availability from the lapping industry, transparency in visible and infrared wavelengths, stability under intense laser light, and chemical and biological inertia under regular biological cultural environment. For applications in the study 
of biophysical properties, these new optical handles can thus provide excess degrees of freedom to manipulate cells and molecular assemblies while avoiding direct contact of light with the biological specimen being studied.

\section{ACKNOWLEDGMENTS}

The authors acknowledge the technical support provided by Wey-Seen Wang. This study was sponsored by the National Science Council, Republic of China, under grant numbers NSC 90-2215-E-002-039 (to C.-K. Sun), NSC 892311-B-001-032 (to B.-L. Lin), and NSC 89-2811-E-0020058 (to P. C. Cheng).

C.-K. Sun's e-mail address is sun@cc.ee.ntu.edu.tw.

\section{REFERENCES}

1. A. Ashkin, J. M. Dziedzic, and T. Yamane, "Optical trapping and manipulation of single cells using infrared laser beams," Nature 330, 769-771 (1987).

2. A. Ashkin and J. M. Dziedzic, "Optical trapping and manipulation of viruses and bacteria," Science 235, 1517-1520 (1987).

3. K. Svoboda and S. M. Block, "Force and velocity measured for single kinesin molecules," Cell 77, 773-784 (1994).

4. J. T. Finer, R. M. Simmons, and J. A. Spudich, "Single myosin molecule mechanics: piconewton forces and nanometer steps," Nature 368, 113-119 (1994)

5. T. T. Perkins, S. R. Quake, D. E. Smith, and S. Chu, "Relaxation of single DNA molecule observed by optical microscopy," Science 264, 822-826 (1994).

6. N. Suzuki, H. Miyata, S. Ishiwata, and K. Kinosita, Jr. "Preparation of bead-tailed actin filaments: estimation of the torque produced by the sliding force in an in vitro motility assay," Biophys. J. 70, 401-408 (1996).

7. M. E. J. Friese, J. Enger, H. Rubinsztein-Dunlop, and N. R. Heckenberg, "Optical angular-momentum transfer to trapped absorbing particles," Phys. Rev. A 54, 1593-1596 (1996).

8. N. B. Simpson, K. Dholakia, L. Allen, and M. J. Padgett, "Mechanical equivalence of spin and orbital angular momentum of light: an optical spanner," Opt. Lett. 22, 52-54 (1997).

9. S. Sato, M. Ishigure, and H. Inaba, "Optical trapping and rotational manipulation of microscopic particles and biological cells using higher-order mode Nd:YAG laser beams," Electron. Lett. 27, 1831-1832 (1991).

10. E. Higurashi, R. Sawada, and T. Ito, "Optical induced angular alignment of birefringent micro-objects by linear polarization,” Appl. Phys. Lett. 73, 3034-3036 (1998).

11. M. E. J. Friese, T. A. Nieminen, N. R. Heckengerg, and H. Rubinsztein-Dunlop, "Optical alignment and spinning of laser-trapped microscopic particles," Nature 394, 348-350 (1998).
12. H. Misawa, K. Sasaki, M. Koshioka, N. Kitamura and H. Masuhara, "Multibeam laser manipulation and fixation of microparticles," Appl. Phys. Lett. 60, 310-312 (1992).

13. Y. G. Song, S. Chang, and J. H. Jo, "Optically induced rotation of combined Mie particles within an evanescent field of a Gaussian beam,” Jpn. J. Appl. Phys., Part 2 38, L380L383 (1999).

14. E. Higurashi, H. Ukita, H. Tanaka, and O. Ohguchi, "Optically induced rotation of isotropic micro-objects fabricated by surface micromachining," Appl. Phys. Lett. 64, 22092210 (1994).

15. R. C. Gauthier, "Theretocal model for an improved radiation pressure micromotor," Appl. Phys. Lett. 69, 2015-2017 (1996).

16. E. Higurashi, O. Ohguchi, T. Tamamura, H. Ukita, and R. Sawada, "Optically induced rotation of dissymmetrically shaped fluorinated polyimide micro-objects in optical traps,” J. Appl. Phys. 82, 2773-2779 (1997).

17. E. Higurashi, R. Sawada, and T. Ito, "Optically induced rotation of a trapped micro-object about an axis perpendicular to the laser beam axis," Appl. Phys. Lett. 72, 2951-2953 (1998).

18. Z.-P. Luo, Y.-L. Sun, and K.-N. An, "An optical spin micromoter," Appl. Phys. Lett. 76, 1779-1781 (2000).

19. A. Yamamoto and I. Yamaguchi, "Measurement and control of optically induced rotation of anisotropic shaped particles,” Jpn. J. Appl. Phys., Part 1 34, 3104-3108 (1995).

20. R. Omori, K. Shima, and A. Suzuki, "Rotation of optically trapped particles in air," Jpn. J. Appl. Phys., Part 2 38, L743-L735 (1999).

21. K. Konig, H. Liang, M. W. Berns, and B. J. Tromberg, "Cell damage in near-infrared multimode optical traps as a result of multiphoton absorption," Opt. Lett. 21, 1090-1092 (1996).

22. K. Konig, H. Liang, M. W. Berns, and B. J. Tromberg, "Cell damage by near-IR microbeams," Nature 377, 20-21 (1995).

23. P. C. Cheng, B.-L. Lin, F.-J. Kao, and C.-K. Sun, "Multiphoton fluorescence microscopy: behavior of biological specimens under high intensity illumination," in Optical Sensing, Imaging and Manipulation for Biological and Biomedical Applications, R. R. Alfano, P.-P. Ho, and A. E. T. Chiou, eds., Proc. SPIE 4082, 134-138 (2000).

24. H. C. Huang and C. C. Chen, "Genome multiplication in cultured protoplasts of two Nicotiana species," J. Hered. 79, 28-32 (1988).

25. B. S. Jacobson and D. Branton, "Plasma membrane: rapid isolation and exposure of the cytoplasmic surface by use of positively charged beads," Science 195, 302-304 (1977).

26. M. W. Berns, J. R. Aist, W. H. Wright, and H. Liang, "Optical trapping in animal and fungal cells using a tunable near-infrared titanium-sapphire laser," Exp. Cell Res. 198, 375-378 (1992).

27. J.-S. Kim and S.-W. Kim, "Dynamic motion analysis of optically trapped nonspherical particles with off-axis position and arbitrary orientation," Appl. Opt. 39, 4327-4332 (2000). 